International Journal of Pure and Applied Mathematics

Volume 104 No. 1 2015, 87-106

ISSN: 1311-8080 (printed version); ISSN: 1314-3395 (on-line version)

url: http://www.ijpam.eu

doi: http://dx.doi.org/10.12732/ijpam.v104i1.8

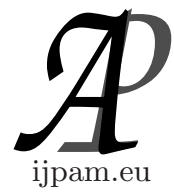

\title{
ANALYSIS AND STUDY OF WEAK SOLUTION OF ANTIPLANE ELECTRO-VISCOELASTIC PROBLEM
}

\author{
Ammar Derbazi ${ }^{1}$, Amar Megrous $^{2}$, Mohamed Dalah ${ }^{3} \S$ \\ ${ }^{1}$ Department of Mathematics \\ Faculty of MI \\ Ammar Derbazi University Bachir El Ibrahimi \\ Bordj Bou Arreridj, 34 000, ALGERIA \\ ${ }^{2}$ Amar Megrous Ecole Préparatoire en Sciences Economiques \\ Commerciales et Sciences de Gestion EPSE-CSG of Constantine \\ 25 000, ALGERIA \\ ${ }^{3}$ Department of Mathematics \\ Faculty of Sciences \\ Mohamed Dalah University of Frères Mentouri Constantine \\ Constantine, 25000, ALGERIA
}

\begin{abstract}
In this work we study a mathematical model which describes the antiplane shear deformation of a cylinder in frictionless contact with a rigid foundation. The material is assumed to be electro-viscoelastic with long-term memory, and the friction is modeled with Tresca's law and the foundation is assumed to be electrically conductive. First we derive the classical variational formulation of the model which is given by a system coupling an evolutionary variational equality for the displacement field and a time-dependent variational equation for the potential field. Then we prove the existence of a unique weak solution to the model. Moreover, the Proof is based on arguments of evolution equations and by using the Banach fixed-point theorem.
\end{abstract}

Received: April 29, 2015

(c) 2015 Academic Publications, Ltd.

${ }^{\S}$ Correspondence author url: www.acadpubl.eu 
AMS Subject Classification: 74M10, 74F15, 74G25, 49J40

Key Words: Tresca's friction, antiplane shears deformation, electro-viscoelastic material, variational inequality, weak solution, fixed point

\section{Introduction}

In this paper we study an antiplane contact problem for electro-viscoelastic materials with long-term memory. We consider the case of antiplane shear deformation i.e., the dispalcement is parallel to the generators of the cylinder and is dependent of the axial coordinate. Our interest is to describe a physical process (for more details see $[1,2,5]$ ) in which both antiplane shear, contact, state of material with long-term memory and piezoelectric effect are involved, leading to a well posedness mathematical problem. In the variational formulation, this kind of problem leads to an integro-differential inequality. The main result we provide concerns the existence of a unique weak solution to the model, see for instance $[2,5,4]$ for details.

The rest of the paper is structured as follows. In Section s:2 we describe the model of the frictional contact process between electro-viscoelastic body and a conductive deformable foundation. In Section s:3 we derive the variational formulation. It consists of a variational inequality for the displacement field coupled with a time-dependent variational equation for the electric potential. We state our main result, the existence of a unique weak solution to the model in Theorem t:1. The Proof of the Theorem is provided in the end of Section s:4, where it is based on arguments of evolutionary inequalities, and a fixed point Theorem.

\section{The Model of the Antiplane Contact Problem}

We consider a piezoelectric body $\mathcal{B}$ identified with a region in $\mathbb{R}^{3}$ it occupies in a fixed and undistorted reference configuration. We assume that $\mathcal{B}$ is a cylinder with generators parallel to the $x_{3}$-axes with a cross-section which is a regular region $\Omega$ in the $x_{1}, x_{2}$-plane, $O x_{1} x_{2} x_{3}$ being a Cartesian coordinate system. The cylinder is assumed to be sufficiently long so that the end effects in the axial direction are negligible. Thus, $\mathcal{B}=\Omega \times(-\infty,+\infty)$. The cylinder is acted upon by body forces of density $\mathbf{f}_{0}$ and has volume free electric charges of density $q_{0}$. It is also constrained mechanically and electrically on the boundary. To describe the boundary conditions, we denote by $\partial \Omega=\Gamma$ the boundary of $\Omega$ and we assume a partition of $\Gamma$ into three open disjoint parts $\Gamma_{1}, \Gamma_{2}$ and $\Gamma_{3}$, on the one 
hand, and a partition of $\Gamma_{1} \cup \Gamma_{2}$ into two open parts $\Gamma_{a}$ and $\Gamma_{b}$, on the other hand. We assume that the one-dimensional measure of $\Gamma_{1}$ and $\Gamma_{a}$, denoted meas $\Gamma_{1}$ and meas $\Gamma_{a}$, are positive. The cylinder is clamped on $\Gamma_{1} \times(-\infty,+\infty)$ and therefore the displacement field vanishes there. Surface tractions of density $\mathbf{f}_{2}$ act on $\Gamma_{2} \times(-\infty,+\infty)$. We also assume that the electrical potential vanishes on $\Gamma_{a} \times(-\infty,+\infty)$ and a surface electrical charge of density $q_{2}$ is prescribed on $\Gamma_{b} \times(-\infty,+\infty)$. The cylinder is in contact over $\Gamma_{3} \times(-\infty,+\infty)$ with a conductive obstacle, the so called foundation. The contact is frictional and is modeled with Tresca's law. We are interested in the deformation of the cylinder on the time interval $[0, T]$. We assume that

$$
\begin{gathered}
\mathbf{f}_{0}=\left(0,0, f_{0}\right) \quad \text { with } f_{0}=f_{0}\left(x_{1}, x_{2}, t\right): \Omega \times[0, T] \rightarrow \mathbb{R}, \\
\mathbf{f}_{2}=\left(0,0, f_{2}\right) \quad \text { with } f_{2}=f_{2}\left(x_{1}, x_{2}, t\right): \Gamma_{2} \times[0, T] \rightarrow \mathbb{R}, \\
q_{0}=q_{0}\left(x_{1}, x_{2}, t\right): \Omega \times[0, T] \rightarrow \mathbb{R}, \\
q_{2}=q_{2}\left(x_{1}, x_{2}, t\right): \Gamma_{b} \times[0, T] \rightarrow \mathbb{R} .
\end{gathered}
$$

The forces (1), (2) and the electric charges (3), (4) would be expected to give rise to deformations and to electric charges of the piezoelectric cylinder corresponding to a displacement $\mathbf{u}$ and to an electric potential field $\varphi$ which are independent on $x_{3}$ and have the form

$$
\begin{gathered}
\mathbf{u}=(0,0, u) \quad \text { with } u=u\left(x_{1}, x_{2}, t\right): \Omega \times[0, T] \rightarrow \mathbb{R}, \\
\varphi=\varphi\left(x_{1}, x_{2}, t\right): \Omega \times[0, T] \rightarrow \mathbb{R} .
\end{gathered}
$$

Such kind of deformation, associated to a displacement field of the form (5), is called an antiplane shear, see for instance $[1,2,4]$ for details.

Below in this paper the indices $i$ and $j$ denote components of vectors and tensors and run from 1 to 3 , summation over two repeated indices is implied, and the index that follows a comma represents the partial derivative with respect to the corresponding spatial variable; also, a dot above represents the time derivative. We use $\mathcal{S}^{3}$ for the linear space of second order symmetric tensors on $\mathbb{R}^{3}$ or, equivalently, the space of symmetric matrices of order 3 , and ".", $\|\cdot\|$ will represent the inner products and the Euclidean norms on $\mathbb{R}^{3}$ and $\mathcal{S}^{3}$; we have :

$$
\begin{gathered}
\mathbf{u} \cdot \mathbf{v}=u_{i} v_{i}, \quad\|\mathbf{v}\|=(\mathbf{v} \cdot \mathbf{v})^{1 / 2} \quad \text { for all } \mathbf{u}=\left(u_{i}\right), \mathbf{v}=\left(v_{i}\right) \in \mathbb{R}^{3} \\
\boldsymbol{\sigma} \cdot \boldsymbol{\tau}=\sigma_{i j} \tau_{i j}, \quad\|\boldsymbol{\tau}\|=(\boldsymbol{\tau} \cdot \boldsymbol{\tau})^{1 / 2} \quad \text { for all } \boldsymbol{\sigma}=\left(\sigma_{i j}\right), \boldsymbol{\tau}=\left(\tau_{i j}\right) \in \mathcal{S}^{3} .
\end{gathered}
$$

The infinitesimal strain tensor is denoted $\varepsilon(\mathbf{u})=\left(\varepsilon_{i j}(\mathbf{u})\right)$ and the stress field by $\boldsymbol{\sigma}=\left(\sigma_{i j}\right)$. We also denote by $\mathbf{E}(\varphi)=\left(E_{i}(\varphi)\right)$ the electric field and by 
$\mathbf{D}=\left(D_{i}\right)$ the electric displacement field. Here and below, in order to simplify the notation, we do not indicate the dependence of various functions on $x_{1}, x_{2}$, $x_{3}$ or $t$ and we recall that

$$
\varepsilon_{i j}(\mathbf{u})=\frac{1}{2}\left(u_{i, j}+u_{j, i}\right), \quad E_{i}(\varphi)=-\varphi,_{i} .
$$

The material's is modeled by the following electro-viscoelastic constitutive law with long-term memory

$$
\begin{gathered}
\boldsymbol{\sigma}=\lambda(\operatorname{tr} \boldsymbol{\varepsilon}(\mathbf{u})) \mathbf{I}+2 \mu \varepsilon(\mathbf{u})+2 \int_{0}^{t} \theta(t-s) \boldsymbol{\varepsilon}(\mathbf{u}(\mathbf{s})) d s-\mathcal{E}^{*} \mathbf{E}(\varphi) \\
\mathbf{D}=\mathcal{E} \boldsymbol{\varepsilon}(\mathbf{u})+\beta \mathbf{E}(\varphi)
\end{gathered}
$$

where $\lambda$ and $\mu$ are the Lamé coefficients, $\theta:[0, T] \longrightarrow \mathbb{R}$ is viscosity coefficient, $\operatorname{tr} \varepsilon(\mathbf{u})=\varepsilon_{i i}(\mathbf{u}), \mathbf{I}$ is the unit tensor in $\mathbb{R}^{3}, \beta$ is the electric permittivity constant, $\mathcal{E}$ represents the third-order piezoelectric tensor and $\mathcal{E}^{*}$ is its transpose. In the antiplane context (5), (6), using the constitutive equations (7), (8) it follows that the stress field and the electric displacement field are given by

$$
\begin{gathered}
\boldsymbol{\sigma}=\left(\begin{array}{ccc}
0 & 0 & \boldsymbol{\sigma}_{13} \\
0 & 0 & \boldsymbol{\sigma}_{23} \\
\boldsymbol{\sigma}_{31} & \boldsymbol{\sigma}_{32} & 0
\end{array}\right) \\
\mathbf{D}=\left(\begin{array}{c}
e u, 1-\beta \varphi, 1 \\
e u, 2-\beta \varphi, 2 \\
0
\end{array}\right)
\end{gathered}
$$

where

$$
\boldsymbol{\sigma}_{13}=\boldsymbol{\sigma}_{31}=\mu \partial_{x_{1}} u+\int_{0}^{t} \theta(t-s) \partial_{x_{1}} u(s) d s
$$

and

$$
\boldsymbol{\sigma}_{23}=\boldsymbol{\sigma}_{32}=\mu \partial_{x_{2}} u+\int_{0}^{t} \theta(t-s) \partial_{x_{2}} u(s) d s .
$$

We assume that

$$
\mathcal{E} \boldsymbol{\varepsilon}=\left(\begin{array}{c}
e\left(\varepsilon_{13}+\varepsilon_{31}\right) \\
e\left(\varepsilon_{23}+\varepsilon_{32}\right) \\
e \varepsilon_{33}
\end{array}\right) \quad \forall \boldsymbol{\varepsilon}=\left(\varepsilon_{i j}\right) \in \mathcal{S}^{3}
$$

where $e$ is a piezoelectric coefficient. We also assume that the coefficients $\theta$, $\mu, \beta$ and $e$ depend on the spatial variables $x_{1}, x_{2}$, but are independent on the 
spatial variable $x_{3}$. Since $\mathcal{E} \varepsilon \cdot \mathbf{v}=\boldsymbol{\varepsilon} \cdot \mathcal{E}^{*} \mathbf{v}$ for all $\varepsilon \in \mathcal{S}^{3}, \mathbf{v} \in \mathbb{R}^{3}$, it follows from (11) that

$$
\mathcal{E}^{*} \mathbf{v}=\left(\begin{array}{ccc}
0 & 0 & e v_{1} \\
0 & 0 & e v_{2} \\
e v_{1} & e v_{2} & e v_{3}
\end{array}\right) \quad \forall \mathbf{v}=\left(v_{i}\right) \in \mathbb{R}^{3}
$$

We assume that the process is mechanically quasistatic and electrically static and therefore is governed by the equilibrium equations

$$
\operatorname{Div} \boldsymbol{\sigma}+\mathbf{f}_{0}=\mathbf{0}, \quad D_{i, i}-q_{0}=0 \quad \text { in } \mathcal{B} \times(0, T),
$$

where $\operatorname{Div} \boldsymbol{\sigma}=\left(\sigma_{i j, j}\right)$ represents the divergence of the tensor field $\boldsymbol{\sigma}$. Taking into account (1), (3), (5), (6), (9) and (10), the equilibrium equations above reduce to the following scalar equations

$$
\begin{gathered}
\operatorname{div}(\mu \nabla u)+\int_{0}^{t} \theta(t-s) \operatorname{div}(\nabla u(s)) d s+\operatorname{div}(e \nabla \varphi)+f_{0}=0, \text { in } \Omega \times(0, T), \\
\operatorname{div}(e \nabla u-\beta \nabla \varphi)=q_{0} .
\end{gathered}
$$

Here and below we use the notation

$$
\begin{gathered}
\operatorname{div} \boldsymbol{\tau}=\tau_{1,1}+\tau_{1,2} \quad \text { for } \boldsymbol{\tau}=\left(\tau_{1}\left(x_{1}, x_{2}, t\right), \tau_{2}\left(x_{1}, x_{2}, t\right)\right), \\
\nabla v=\left(v_{, 1}, v_{, 2}\right), \quad \partial_{\nu} v=v,_{1} \nu_{1}+v, \nu_{2} \quad \text { for } v=v\left(x_{1}, x_{2}, t\right) .
\end{gathered}
$$

We now describe the boundary conditions. During the process the cylinder is clamped on $\Gamma_{1} \times(-\infty,+\infty)$ and the electric potential vanish on $\Gamma_{1} \times$ $(-\infty,+\infty)$; thus, (5) and (6) imply that

$$
\begin{array}{ll}
u=0 & \text { on } \Gamma_{1} \times(0, T), \\
\varphi=0 & \text { on } \Gamma_{a} \times(0, T) .
\end{array}
$$

Let $\boldsymbol{\nu}$ denote the unit normal on $\Gamma \times(-\infty,+\infty)$. We have

$$
\boldsymbol{\nu}=\left(\nu_{1}, \nu_{2}, 0\right) \quad \text { with } \nu_{i}=\nu_{i}\left(x_{1}, x_{2}\right): \Gamma \rightarrow \mathbb{R}, \quad i=1,2 .
$$

For a vector $\mathbf{v}$ we denote by $v_{\nu}$ and $\mathbf{v}_{\tau}$ its normal and tangential components on the boundary, given by

$$
v_{\nu}=\mathbf{v} \cdot \boldsymbol{\nu}, \quad \mathbf{v}_{\tau}=\mathbf{v}-v_{\nu} \boldsymbol{\nu}
$$


For a given stress field $\sigma$ we denote by $\sigma_{\nu}$ and $\sigma_{\tau}$ the normal and the tangential components on the boundary, that is

$$
\sigma_{\nu}=(\boldsymbol{\sigma} \nu) \cdot \boldsymbol{\nu}, \quad \boldsymbol{\sigma}_{\tau}=\boldsymbol{\sigma} \boldsymbol{\nu}-\sigma_{\nu} \boldsymbol{\nu}
$$

From (9), (10) and (17) we deduce that the Cauchy stress vector and the normal component of the electric displacement field are given by

$$
\boldsymbol{\sigma} \boldsymbol{\nu}=\left(0,0, \mu \partial_{\nu} u+\int_{0}^{t} \theta(t-s) \partial_{\nu} u(s) d s+e \partial_{\nu} \varphi\right), \quad \mathbf{D} \cdot \boldsymbol{\nu}=e \partial_{\nu} u-\beta \partial_{\nu} \varphi
$$

Taking into account (2), (4) and (20), the traction condition on $\Gamma_{2} \times$ $(-\infty, \infty)$ and the electric conditions on $\Gamma_{b} \times(-\infty, \infty)$ are given by

$$
\begin{gathered}
\mu \partial_{\nu} u+\int_{0}^{t} \theta(t-s) \partial_{\nu} u(s) d s+e \partial_{\nu} \varphi=f_{2} \quad \text { on } \Gamma_{2} \times(0, T), \\
e \partial_{\nu} u-\beta \partial_{\nu} \varphi=q_{2} \quad \text { on } \Gamma_{b} \times(0, T) .
\end{gathered}
$$

We now describe the frictional contact condition and the electric conditions on $\Gamma_{3} \times(-\infty,+\infty)$. First, from (5) and (17) we infer that the normal displacement vanishes, $u_{\nu}=0$, which shows that the contact is bilateral, that is, the contact is kept during all the process. Using now (5) and (17)-(19) we conclude that

$$
\mathbf{u}_{\tau}=(0,0, u), \quad \boldsymbol{\sigma}_{\tau}=\left(0,0, \sigma_{\tau}\right)
$$

where

$$
\sigma_{\tau}=\left(0,0, \mu \partial_{\nu} u+\int_{0}^{t} \theta(t-s) \partial_{\nu} u(s) d s+e \partial_{\nu} \varphi\right)
$$

We assume that the friction is invariant with respect to the $x_{3}$ axis and is modeled with Tresca's friction law, that is

$$
\left\{\begin{array}{l}
\left|\boldsymbol{\sigma}_{\tau}(t)\right| \leq g, \\
\left|\boldsymbol{\sigma}_{\tau}(t)\right|<g \Rightarrow \dot{\boldsymbol{u}}_{\tau}(t)=\mathbf{0}, \quad \text { on } \Gamma_{3} \times(0, T) . \\
\left|\boldsymbol{\sigma}_{\tau}(t)\right|=g \Rightarrow \exists \beta \geq 0 \text { such that } \boldsymbol{\sigma}_{\tau}=-\beta \dot{\boldsymbol{u}}_{\tau}
\end{array}\right.
$$

Here $g: \Gamma_{3} \rightarrow \mathbb{R}_{+}$is a given function, the friction bound, and $\dot{\mathbf{u}}_{\tau}$ represents the tangential velocity on the contact boundary, see $[2,5]$ for details. Using now 
(23) it is straightforward to see that the friction law (24) implies

$$
\left\{\begin{array}{l}
\left|\mu \partial_{\nu} u+\int_{0}^{t} \theta(t-s) \partial_{\nu} u(s) d s+e \partial_{\nu} \varphi\right| \leq g, \\
\left|\mu \partial_{\nu} u+\int_{0}^{t} \theta(t-s) \partial_{\nu} u(s) d s+e \partial_{\nu} \varphi\right|<g \Rightarrow \dot{u}(t)=0, \text { on } \Gamma_{3} \times(0, T) . \\
\left|\mu \partial_{\nu} u+\int_{0}^{t} \theta(t-s) \partial_{\nu} u(s) d s+e \partial_{\nu} \varphi\right|=g \Rightarrow \exists \beta \geq 0 \quad \text { such that } \\
\mu \partial_{\nu} u+\int_{0}^{t} \theta(t-s) \partial_{\nu} u(s) d s+e \partial_{\nu} \varphi=-\beta \dot{u}
\end{array}\right.
$$

Next, since the foundation is electrically conductive and the contact is bilateral, we assume that the normal component of the electric displacement field or the free charge is proportional to the difference between the potential on the foundation and the body's surface. Thus,

$$
\mathbf{D} \cdot \boldsymbol{\nu}=k\left(\varphi-\varphi_{F}\right) \quad \text { on } \quad \Gamma_{3} \times(0, T),
$$

where $\varphi_{F}$ represents the electric potential of the foundation and $k$ is the electric conductivity coefficient. We use (20) and the previous equality to obtain

$$
e \partial_{\nu} u-\beta \partial_{\nu} \varphi=k\left(\varphi-\varphi_{F}\right) \text { on } \Gamma_{3} \times(0, T) .
$$

Finally, we prescribe the initial displacement,

$$
u(0)=u_{0} \quad \text { in } \Omega,
$$

where $u_{0}$ is a given function on $\Omega$.

We collect the above equations and conditions to obtain the following mathematical model which describes the antiplane shear of an electro-viscoelastic cylinder in frictional contact with a conductive foundation.

\section{Problem P}

Find the displacement field $u: \Omega \times[0, T] \rightarrow \mathbb{R}$ and the electric potential $\varphi$ : $\Omega \times[0, T] \rightarrow \mathbb{R}$ such that

$$
\operatorname{div}(\mu \nabla u)+\int_{0}^{t} \theta(t-s) \operatorname{div}(\nabla u(s)) d s+\operatorname{div}(e \nabla \varphi)+f_{0}=0 \quad \text { in } \Omega \times(0, T),
$$

$$
\operatorname{div}(e \nabla u-\alpha \nabla \varphi)=q_{0} \quad \text { in } \Omega \times(0, T)
$$




$$
\begin{gathered}
u=0 \quad \text { on } \Gamma_{1} \times(0, T) \\
\mu \partial_{\nu} u+\int_{0}^{t} \theta(t-s) \partial_{\nu} u(s) d s+e \partial_{\nu} \varphi=f_{2} \quad \text { on } \Gamma_{2} \times(0, T), \\
\left\{\begin{array}{c}
\left|\mu \partial_{\nu} u+\int_{0}^{t} \theta(t-s) \partial_{\nu} u(s) d s+e \partial_{\nu} \varphi\right| \leq g, \\
\left|\mu \partial_{\nu} u+\int_{0}^{t} \theta(t-s) \partial_{\nu} u(s) d s+e \partial_{\nu} \varphi\right|<g \Rightarrow \dot{u}(t)=0, \text { on } \Gamma_{3} \times(0, T), \\
\left|\mu \partial_{\nu} u+\int_{0}^{t} \theta(t-s) \partial_{\nu} u(s) d s+e \partial_{\nu} \varphi\right|=g \Rightarrow \exists \beta 00 \text { such that } \\
\mu \partial_{\nu} u+\int_{0}^{t} \theta(t-s) \partial_{\nu} u(s) d s+e \partial_{\nu} \varphi=-\beta \dot{u}, \\
e \partial_{\nu} u-\alpha \partial_{\nu} \varphi=q_{2} \quad \text { on } \Gamma_{b} \times(0, T), \\
e \partial_{\nu} u-\alpha \partial_{\nu} \varphi=k(\varphi-\varphi) \quad \text { on } \Gamma_{3} \times(0, T),
\end{array}\right.
\end{gathered}
$$

Note that once the displacement field $u$ and the electric potential $\varphi$ which solve Problem $\mathcal{P}$ are known, then the stress tensor $\boldsymbol{\sigma}$ and the electric displacement field $\mathbf{D}$ can be obtained by using the constitutive laws (9) and (10), respectively.

\section{Variational Formulation and Main Result}

We derive now the variational formulation of the Problem $\mathcal{P}$. To this end we introduce the function spaces

$$
V=\left\{v \in H^{1}(\Omega): v=0 \text { on } \Gamma_{1}\right\}, \quad W=\left\{\psi \in H^{1}(\Omega): \psi=0 \text { on } \Gamma_{a}\right\}
$$


where, here and below, we write $w$ for the trace $\gamma w$ of a function $w \in H^{1}(\Omega)$ on $\Gamma$. Since meas $\Gamma_{1}>0$ and meas $\Gamma_{a}>0$, it is well known that $V$ and $W$ are real Hilbert spaces with the inner products

$$
(u, v)_{V}=\int_{\Omega} \nabla u \cdot \nabla v d x \quad \forall u, v \in V, \quad(\varphi, \psi)_{W}=\int_{\Omega} \nabla \varphi \cdot \nabla \psi d x \quad \forall \varphi, \psi \in W .
$$

Moreover, the associated norms

$$
\|v\|_{V}=\|\nabla v\|_{L^{2}(\Omega)^{2}} \quad \forall v \in V, \quad\|\psi\|_{W}=\|\nabla \psi\|_{L^{2}(\Omega)^{2}} \quad \forall \psi \in W
$$

are equivalent on $V$ and $W$, respectively, with the usual norm $\|\cdot\|_{H^{1}(\Omega)}$. By Sobolev's trace theorem we deduce that there exist two positive constants $c_{V}>$ 0 and $c_{W}>0$ such that

$$
\|v\|_{L^{2}\left(\Gamma_{3}\right)} \leq c_{V}\|v\|_{V} \quad \forall v \in V, \quad\|\psi\|_{L^{2}\left(\Gamma_{3}\right)} \leq c_{W}\|\psi\|_{W} \quad \forall \psi \in W .
$$

For a real Banach space $\left(X,\|\cdot\|_{X}\right)$ we use the usual notation for the spaces $L^{p}(0, T ; X)$ and $W^{k, p}(0, T ; X)$ where $1 \leq p \leq \infty, k=1,2, \ldots$; we also denote by $C([0, T] ; X)$ the space of continuous and continuously differentiable functions on $[0, T]$ with values in $X$, with the norm

$$
\|x\|_{C([0, T] ; X)}=\max _{t \in[0, T]}\|x(t)\|_{X}
$$

and we use the standart notations for the Lebesgue space $L^{2}(0, T ; X)$ as well as the Sobolev space $W^{1,2}(0, T ; X)$. In particular, recall that the norm on the space $L^{2}(0, T ; X)$ is given by the formula

$$
\|u\|_{L^{2}(0, T ; X)}^{2}=\int_{0}^{T}\|u(t)\|_{X}^{2} d t
$$

and the norm on the space $W^{2}(0, T ; X)$ is given by the formula

$$
\|u\|_{W^{1,2}(0, T ; X)}^{2}=\int_{0}^{T}\|u(t)\|_{X}^{2} d t+\int_{0}^{T}\|\dot{u}(t)\|_{X}^{2} d t .
$$

Finally, we suppose the argument $X$ when $X=\mathbb{R}$; thus, for example, we use the notation $W^{2}(0, T)$ for the space $W^{2}(0, T ; \mathbb{R})$ and the notation $\|\cdot\|_{W^{2}(0, T)}$ for the norm $\|\cdot\|_{W^{2}(0, T ; \mathbb{R})}$.

In the study of the Problem $\mathcal{P}$ we assume that the viscosity coefficient satisfy

$$
\theta \in W^{1,2}(0, T)
$$


and the electric permittivity coefficient satisfy

$\alpha \in L^{\infty}(\Omega)$ and there exists $\alpha^{*}>0$ such that $\alpha(\mathbf{x}) \geq \alpha^{*}$ a.e. $\mathbf{x} \in \Omega$.

We also assume that the Lamé coefficient and the piezoelectric coefficient satisfy

$$
\begin{gathered}
\mu \in L^{\infty}(\Omega) \text { and } \mu(\mathbf{x})>0 \text { a.e. } \mathbf{x} \in \Omega, \\
e \in L^{\infty}(\Omega) .
\end{gathered}
$$

The forces, tractions, volume and surface free charge densities have the regularity

$$
\begin{array}{ll}
f_{0} \in W^{1,2}\left(0, T ; L^{2}(\Omega)\right), & f_{2} \in W^{1,2}\left(0, T ; L^{2}\left(\Gamma_{2}\right)\right), \\
q_{0} \in W^{1,2}\left(0, T ; L^{2}(\Omega)\right), & q_{2} \in W^{1,2}\left(0, T ; L^{2}\left(\Gamma_{b}\right)\right) .
\end{array}
$$

The electric conductivity coefficient and the friction bound function $g$ satisfies the following properties

$$
\begin{aligned}
& k \in L^{\infty}\left(\Gamma_{3}\right) \text { and } k(\mathbf{x}) \geq 0 \text { a.e. } \mathbf{x} \in \Gamma_{3} \text {, } \\
& g \in L^{\infty}\left(\Gamma_{3}\right) \text { and } g(\mathbf{x}) \geq 0 \text { a.e. } \mathbf{x} \in \Gamma_{3} \text {. }
\end{aligned}
$$

Finally, we assume that the electric potential of the foundation and the initial displacement are such that

$$
\varphi_{F} \in W^{1,2}\left(0, T ; L^{2}\left(\Gamma_{3}\right)\right) .
$$

The initial data are chosen such that

$$
u_{0} \in V
$$

and, moreover,

$$
a_{\mu}\left(u_{0}, v\right)_{V}+j(v) \geq(f(0), v)_{V} \quad \forall v \in V .
$$

We define now the functional $j:[0, T] \longrightarrow \mathbb{R}_{+}$given by the formula

$$
j(v)=\int_{\Gamma_{3}} g|v| d a \quad \forall v \in V .
$$

We also define the mappings $f:[0, T] \rightarrow V$ and $q:[0, T] \rightarrow W$, respectively, by

$$
(f(t), v)_{V}=\int_{\Omega} f_{0}(t) v d x+\int_{\Gamma_{2}} f_{2}(t) v d a
$$




$$
(q(t), \psi)_{W}=\int_{\Omega} q_{0}(t) \psi d x-\int_{\Gamma_{b}} q_{2}(t) \psi d a+\int_{\Gamma_{3}} k \varphi_{F}(t) \psi d a,
$$

for all $v \in V, \psi \in W$ and $t \in[0, T]$. The definition of $f$ and $q$ are based on Riesz's representation theorem; moreover, it follows from assumptions by (42)-(43), that the integrals above are well-defined and

$$
\begin{aligned}
& f \in W^{1,2}(0, T ; V), \\
& q \in W^{1,2}(0, T ; W) .
\end{aligned}
$$

Next, we define the bilinear forms $a_{\mu}: V \times V \rightarrow \mathbb{R}, a_{e}: V \times W \rightarrow \mathbb{R}$, $a_{e}^{*}: W \times V \rightarrow \mathbb{R}$, and $a_{\alpha}: W \times W \rightarrow \mathbb{R}$, by equalities

$$
\begin{gathered}
a_{\mu}(u, v)=\int_{\Omega} \mu \nabla u \cdot \nabla v d x, \\
a_{e}(u, \varphi)=\int_{\Omega} e \nabla u \cdot \nabla \varphi d x=a_{e}^{*}(\varphi, u), \\
a_{\alpha}(\varphi, \psi)=\int_{\Omega} \beta \nabla \varphi \cdot \nabla \psi d x+\int_{\Gamma_{3}} k \varphi \psi d x,
\end{gathered}
$$

for all $u, v \in V, \varphi, \psi \in W$. Assumptions (49)-(51) imply that the integrals above are well defined and, using (36) and (37), it follows that the forms $a_{\mu}$, $a_{e}$ and $a_{e}^{*}$ are continuous; moreover, the forms $a_{\mu}$ and $a_{\alpha}$ are symmetric and, in addition, the form $a_{\alpha}$ is $W$-elliptic, since

$$
a_{\alpha}(\psi, \psi) \geq \alpha^{*}\|\psi\|_{W}^{2} \quad \forall \psi \in W .
$$

The variational formulation of Problem $\mathcal{P}$ is based on the following result.

Lemma 1. If $(u, \varphi)$ is a smooth solution to Problem $\mathcal{P}$, then $(u(t), \varphi(t)) \in$ $X$ and

$$
\begin{gathered}
a_{\mu}(u(t), v-\dot{u}(t))+\left(\int_{0}^{t} \theta(t-s) u(s) d s, v-\dot{u}(t)\right)_{V}+a_{e}^{*}(\varphi(t), v-\dot{u}(t)) \\
+j(v)-j(\dot{u}(t)) \geq(f(t), v-\dot{u}(t))_{V} \quad \forall v \in V, t \in[0, T], \\
a_{\alpha}(\varphi(t), \psi)-a_{e}(u(t), \psi)=(q(t), \psi)_{W} \quad \forall \psi \in W, t \in[0, T], \\
u(0)=u_{0} .
\end{gathered}
$$

Proof. Let $(u, \varphi)$ denote a smooth solution to Problem $\mathcal{P}$, we have $u(t) \in V$, 
$\dot{u}(t) \in V$ and $\varphi(t) \in W$ a.e. $t \in[0, T]$ and, from (28), (30) and (31), we obtain

$$
\begin{aligned}
& \int_{\Omega} \mu \nabla u(t) \cdot \nabla(v-\dot{u}(t)) d x+\left(\int_{0}^{t} \theta(t-s) u(s) d s, v-\dot{u}(t)\right)_{V}+ \\
& \int_{\Omega} e \nabla \varphi(t) \cdot \nabla(v-\dot{u}(t)) d x=\int_{\Omega} f_{0}(t)(v-\dot{u}(t)) d x+\int_{\Gamma_{2}} f_{2}(t)(v-\dot{u}(t)) d a+ \\
& \int_{\Gamma_{3}}\left(\left|\mu \partial_{\nu} u(t)+\int_{0}^{t} \theta(t-s) \partial_{\nu} u(s) d s+e \partial_{\nu} \varphi(t)\right|\right)(v-\dot{u}(t)) d a,
\end{aligned}
$$

$\forall v \in V \quad t \in(0, T)$, and from (29) and (33)-(34) we obtain too

$$
\begin{aligned}
& \int_{\Omega} \alpha \nabla \varphi(t) \cdot \nabla \psi d x-\int_{\Omega} e \nabla u(t) \cdot \nabla \psi d x=\int_{\Omega} q_{0}(t) \psi d x-\int_{\Gamma_{b}} q_{2}(t) \psi d a+ \\
& \int_{\Gamma_{3}} k \varphi_{F}(t) \psi d a, \quad \forall \psi \in W \quad t \in(0, T) .
\end{aligned}
$$

Using (49) and (32) we obtain

$$
\begin{aligned}
& a_{\mu}(u(t), v-\dot{u}(t))+\left(\int_{0}^{t} \theta(t-s) u(s) d s, v-\dot{u}(t)\right)_{V}+a_{e}^{*}(\varphi(t), v-\dot{u}(t))- \\
& \int_{\Gamma_{3}}\left(\left|\mu \partial_{\nu} u(t)+\int_{0}^{t} \theta(t-s) \partial_{\nu} u(s) d s+e \partial_{\nu} \varphi(t)\right|\right)(v-\dot{u}(t)) d a= \\
& (f(t), v-\dot{u}(t))_{V}, \quad \forall v \in V, t \in[0, T] .
\end{aligned}
$$

Keeping in mind (51) and (55)-(56), we find the second equality in Lemma 1, i.e.,

$$
a_{\alpha}(\varphi(t), \psi)-a_{e}(u(t), \psi)=(q(t), \psi)_{W} \quad \forall \psi \in W, t \in[0, T] .
$$

Using the frictional contact condition (32) and (49) on $\Gamma_{3} \times(0, T)$, we deduce that for all $t \in[0, T]$

$$
j(\dot{u}(t))=-\int_{\Gamma_{3}}\left(\left|\mu \partial_{\nu} u(t)+\int_{0}^{t} \theta(t-s) \partial_{\nu} u(s) d s+e \partial_{\nu} \varphi(t)\right|\right) \dot{u}(t) d a,
$$

it's very clearly to see that

$$
j(v) \geq-\int_{\Gamma_{3}}\left(\left|\mu \partial_{\nu} u(t)+\int_{0}^{t} \theta(t-s) \partial_{\nu} u(s) d s+e \partial_{\nu} \varphi(t)\right|\right) v d a, \quad \forall v \in V .
$$

The first inequality in Lemma 1 follows now from (62) and (64)-(65).

Now, from Lemma 1 and condition (60) lead to give the following variational Problem : 


\section{Problem P V}

Find a displacement field $u:[0, T] \rightarrow V$ and an electric potential field $\varphi$ : $[0, T] \rightarrow W$ such that

$$
\begin{gathered}
a_{\mu}(u(t), v-\dot{u}(t))+\left(\int_{0}^{t} \theta(t-s) u(s) d s, v-\dot{u}(t)\right)_{V}+a_{e}^{*}(\varphi(t), v-\dot{u}(t))+ \\
j(v)-j(\dot{u}(t)) \geq(f(t), v-\dot{u}(t))_{V}, \quad \forall v \in V, t \in[0, T], \\
a_{\alpha}(\varphi(t), \psi)-a_{e}(u(t), \psi)=(q(t), \psi)_{W}, \quad \forall \psi \in W, t \in[0, T], \\
u(0)=u_{0} .
\end{gathered}
$$

Our main existence and uniqueness result, which we state now and prove in the next section, is the following :

Theorem 2. Assume that (38)-(53) hold. Then the variational problem $\mathcal{P V}$ possesses a unique solution $(u, \varphi)$ satisfies

$$
u \in W^{1,2}(0, T ; V), \quad \varphi \in W^{1,2}(0, T ; W) .
$$

We note that an element $(u, \varphi)$ which solves Problem $\mathcal{P} \mathcal{V}$ is called a weak solution of the antiplane contact Problem $\mathcal{P}$. We conclude by Theorem 2 that the antiplane contact Problem $\mathcal{P}$ has a unique weak solution, provided that (38)-(53) hold.

\section{Proof of Theorem 2}

We start with the proof of Theorem 2 which will be carried out in several steps. To this end, in the rest of this section we assume that (38)-(53) hold.

\section{- Step 1 :}

In the first step of the proof we introduce the set

$$
\mathcal{W}=\left\{\eta \in W^{1,2}(0, T ; X) \text { such that } \eta(0)=0_{X}\right\}
$$

and we recall the following existence and uniquness result.

Lemma 3. For all $\eta \in \mathcal{W}$ there exists a unique element $\eta \in W^{1,2}(0, T ; X)$ such that satisfy the inequality and the data condition defined by the problem $\mathcal{P} \mathcal{V}_{\eta}^{1}$ : 


\section{Problem $\mathrm{P} \mathrm{V}_{\eta}^{1}$}

$$
\begin{gathered}
a\left(u_{\eta}(t), v-\dot{u}_{\eta}(t)\right)+\left(\eta(t), v-\dot{u}_{\eta}(t)\right)_{X}+ \\
j(v)-j\left(\dot{u}_{\eta}(t)\right) \geq\left(f(t), v-\dot{u}_{\eta}(t)\right)_{X}, \quad \forall v \in X, t \in[0, T], \\
u_{\eta}(0)=u_{0} .
\end{gathered}
$$

We use in subsequent demonstration of the previous lemma the following theorem

Theorem 4. ([4], p. 117) Let $\left(X,(\cdot, \cdot)_{X}\right)$ be a real Hilbert space and let $j: X \longrightarrow(-\infty,+\infty)$ be a convex lower semicontinuous functional. Assume that $j \neq+\infty$, that is

$$
D(j)=\{v \in X \quad j(v)<\infty\} \neq \phi .
$$

Let $f \in W^{1,2}(0, T ; X)$ and $u_{0} \in X$ be such that

$$
\sup _{v \in D(j)}=\left\{(f(0), v)_{X}-\left(u_{0}, v\right)_{X}-j(v)\right\}<+\infty .
$$

Then the variational problem $\mathcal{P} \mathcal{V}$ possesses a unique solution $(u, \varphi)$ satisfies $u(0)=u_{0}$ and

$$
(u(t), v-\dot{u}(t))_{X}+j(v)-j(\dot{u}(t)) \leq(f(t), v-\dot{u}(t))_{X} \quad \forall v \in X \quad \text { a.e. } t \in(0, T) .
$$

\section{proof of Lemma 3}

Let $a(\cdot, \cdot)$ define by

$$
a(u, v)=(u, v)_{a} \quad \forall u, v \in X
$$

Notice that $(\cdot, \cdot)_{a}$ is an inner product on the space $X$ and $\|\cdot\|_{a}$ is the associated norm which is equivalent to the norm $\|\cdot\|_{X}$ on the space $X$. Then $\left(X,(\cdot, \cdot)_{a}\right)$ is a real Hilbert space.

We define now the function $f_{\eta}:[0, T] \longrightarrow X$ by the formula

$$
\left(f_{\eta}(t), v\right)_{a}=(f(t), v)_{X}-(\eta(t), v)_{X} \quad \forall v \in V, \quad t \in[0, T] .
$$

It follows from (52) and (70) that

$$
f_{\eta}(t) \in W^{1,2}(0, T ; X) .
$$


Using now (74) at $t=0$, we obtain

$$
\left(f_{\eta}(0), v\right)_{a}=(f(0), v)_{X}-(\eta(0), v)_{X} \quad \forall v \in V, \quad t \in[0, T] .
$$

Moreover, rewriting (73) at $t=0$, we have

$$
a\left(u_{0}, v\right)=\left(u_{0}, v\right)_{a} \quad \forall v \in X
$$

On the other hand, taking into account (70), (76) and (77), we obtain the equality

$$
\left(f_{\eta}(0), v\right)_{a}-\left(u_{0}, v\right)_{a}-j(v)=(f(0), v)_{X}-a\left(u_{0}, v\right)-j(v) \quad \forall v \in V .
$$

From assumption (48), we find

$$
\sup _{v \in D(j)}=\left\{\left(f_{\eta}(0), v\right)_{a}-\left(u_{0}, v\right)_{a}-j(v)\right\}<+\infty .
$$

Taking into account (48), (49), (75) and (79), we can use Theorem 4 on the space $\left(X,(\cdot, \cdot)_{a}\right)$, then there exists a unique element $u_{\eta}$ satisfy

$$
u_{\eta} \in W^{1,2}(0, T ; X) \text { such that } u_{\eta}=u_{0}
$$

and

$$
\begin{gathered}
\left(u_{\eta}(t), v-\dot{u}_{\eta}(t)\right)_{a}+j(v)-j\left(\dot{u}_{\eta}(t)\right) \geq\left(f_{\eta}(t), v-\dot{u}_{\eta}(t)\right)_{a} \\
\forall v \in X \quad \text { a.e. } \quad t \in(0, T) .
\end{gathered}
$$

Using (73) and (76), we obtain the first inequality (71) and the initial data (72) defined in Lemma 3. This concludes the existence and uniqueness part of the proof of Lemma 3 .

\section{- Step 2 :}

In the second step, we use the displacement field $u_{\eta}$ obtained in Lemma 3 to define the following variational Problem for the electric potential field.

\section{Problem $\mathrm{P} \mathrm{V}_{\eta}^{2}$}

Find an electrical potential $\varphi_{\eta}:[0, T] \rightarrow W$ such that

$$
a_{\alpha}\left(\varphi_{\eta}(t), \psi\right)-a_{e}\left(u_{\eta}(t), \psi\right)=(q(t), \psi)_{W} \quad \forall \psi \in W, t \in[0, T] .
$$

The well-posedness of problem $\mathcal{P} \mathcal{V}_{\eta}^{2}$ follows. 
Lemma 5. There exists a unique solution $\varphi_{\eta} \in W^{1,2}(0, T ; W)$ which satisfies (83). Moreover, if $\varphi_{\eta_{1}}$ and $\varphi_{\eta_{2}}$ are the solutions of (83) corresponding to $\eta_{1}, \eta_{2} \in C([0, T] ; V)$ then, there exists $c>0$, such that

$$
\left\|\varphi_{\eta_{1}}(t)-\varphi_{\eta_{2}}(t)\right\|_{W} \leq c\left\|u_{\eta_{1}}(t)-u_{\eta_{2}}(t)\right\|_{V} \quad \forall t \in[0, T] .
$$

Proof. Let $t \in[0, T]$. We use the properties of the bilinear form $a_{\beta}$ and the Lax-Milgram lemma to see that there exists a unique element $\varphi_{\eta}(t) \in W$ which solves (83) at any moment $t \in[0, T]$. Consider now $t_{1}, t_{2} \in[0, T]$; using (83), we get

$$
a_{\alpha}\left(\varphi_{\eta}\left(t_{1}\right), \psi\right)-a_{e}\left(u_{\eta}\left(t_{1}\right), \psi\right)=\left(q\left(t_{1}\right), \psi\right)_{W} \quad \forall \psi \in W, t_{1} \in[0, T]
$$

and

$$
a_{\alpha}\left(\varphi_{\eta}\left(t_{2}\right), \psi\right)-a_{e}\left(u_{\eta}\left(t_{2}\right), \psi\right)=\left(q\left(t_{2}\right), \psi\right)_{W} \quad \forall \psi \in W, t_{2} \in[0, T] .
$$

Using and (84), (85) and (57) we find that

$$
\begin{aligned}
& \alpha^{*}\left\|\varphi\left(t_{1}\right)-\varphi\left(t_{2}\right)\right\|_{W}^{2} \\
& \quad \leq\left(\|e\|_{L^{\infty}(\Omega)}\left\|u\left(t_{1}\right)-u\left(t_{2}\right)\right\|_{V}+\left\|q\left(t_{1}\right)-q\left(t_{2}\right)\right\|_{W}\right)\left\|\varphi\left(t_{1}\right)-\varphi\left(t_{2}\right)\right\|_{W}
\end{aligned}
$$

it follows from the previous inequality that

$$
\left\|\varphi\left(t_{1}\right)-\varphi\left(t_{2}\right)\right\|_{W} \leq c\left(\left\|u\left(t_{1}\right)-u\left(t_{2}\right)\right\|_{V}+\left\|q\left(t_{1}\right)-q\left(t_{2}\right)\right\|_{W}\right) .
$$

Then, the regularity $u_{\eta} \in W^{1,2}(0, T ; V)$ combined with (53) and (86) imply that $\varphi_{\eta} \in W^{1,2}(0, T ; W)$ which concludes the proof.

Now, for all $\eta \in \mathcal{W}$ we denote by $u_{\eta}$ the solution of Problem $\mathcal{P} \mathcal{V}_{\eta}^{1}$ obtained in Lemma 3 and by $\varphi_{\eta}$ the solution of Problem $\mathcal{P} \mathcal{V}_{\eta}^{2}$ obtained in Lemma 5.

\section{- Step 3 :}

In the third step, we consider the operator $\Lambda: \mathcal{W} \longrightarrow \mathcal{W}$.

We now use Riesz's representation theorem to define the element $\Lambda \eta(t) \in \mathcal{W}$ by equality

$$
\begin{gathered}
(\Lambda \eta(t), w)_{\mathcal{W}}=\int_{0}^{t} \theta(t-s) u_{\eta}(s) d s+a_{e}^{*}\left(\varphi_{\eta}(t), w\right), \\
\forall \eta \in \mathcal{W}, \quad \forall w \in W \quad t \in[0, T] .
\end{gathered}
$$

Clearly, for a given $\eta \in \mathcal{W}$ the function $t \mapsto \Lambda \eta(t)$ belongs to $\mathcal{W}$. In this step we show that the operator $\Lambda: \mathcal{W} \rightarrow \mathcal{W}$ has unique fixed point. 
Lemma 6. There exists a unique $\eta^{*} \in \mathcal{W}$ such that $\Lambda \eta^{*}=\eta^{*}$.

Proof. Let $\eta_{1}, \eta_{2} \in \mathcal{W}$ and $t \in[0, T]$. In what follows we denote by $u_{i}$ and $\varphi_{i}$ the functions $u_{\eta_{i}}$ and $\varphi_{\eta_{i}}$ obtained in Lemmas 3 and 5 , for $i=1,2$. Using (87) and (55) we obtain

$$
\begin{gathered}
\left\|\Lambda \eta_{1}(t)-\Lambda \eta_{2}(t)\right\|_{X}^{2} \leq C\left(\int_{0}^{t}\left\|u_{1}(s)-u_{2}(s)\right\|_{X}^{2} d s+\left\|\varphi_{1}(t)-\varphi_{2}(t)\right\|_{W}^{2}\right), \\
\forall t \in[0, T] .
\end{gathered}
$$

The constant $C$ represents a generic positive number which may depend on $\|\theta\|_{W^{1,2}(0, T)}, T$ and $e$, and whose value may change from place to place.

Since $u_{\eta} \in W^{1,2}(0, T ; V)$ and $\varphi_{\eta} \in W^{1,2}(0, T ; W)$ we deduce from inequality (88) that $\Lambda \eta \in W^{1,2}(0, T ; X)$. On the other hand, (83) and arguments similar as those used in the proof of (86) yield

$$
\left\|\varphi_{1}(t)-\varphi_{2}(t)\right\|_{W} \leq C\left\|u_{1}(t)-u_{2}(t)\right\|_{V} .
$$

Using now (89) in (88), we get

$$
\begin{gathered}
\left\|\Lambda \eta_{1}(t)-\Lambda \eta_{2}(t)\right\|_{X}^{2} \leq C\left(\int_{0}^{t}\left\|u_{1}(s)-u_{2}(s)\right\|_{X}^{2} d s+\left\|u_{1}(t)-u_{2}(t)\right\|_{V}\right), \\
\text { a.e.t } \in[0, T] .
\end{gathered}
$$

Taking into account (71) in Lemma 3, we have the inequality

$$
\begin{aligned}
& a\left(u_{1}(t), v-\dot{u}_{1}(t)\right)+\left(\eta_{1}(t), v-\dot{u}_{1}(t)\right)_{X}+ \\
& j(v)-j\left(\dot{u}_{1}(t)\right) \geq\left(f(t), v-\dot{u}_{1}(t)\right)_{X}, \quad \forall v \in X, t \in[0, T]
\end{aligned}
$$

and

$$
\begin{aligned}
& a\left(u_{2}(t), v-\dot{u}_{2}(t)\right)+\left(\eta_{2}(t), v-\dot{u}_{2}(t)\right)_{X}+ \\
& j(v)-j\left(\dot{u}_{2}(t)\right) \geq\left(f(t), v-\dot{u}_{2}(t)\right)_{X}, \quad \forall v \in X, t \in[0, T],
\end{aligned}
$$

for all $v \in X$, a.e.s $s \in(0, T)$. We choose $v=\dot{u}_{2}(s)$ in the first inequality $v=\dot{u}_{1}(s)$ in thes second inequality, add the result to obtain

$$
\frac{1}{2}\left\|u_{1}(s)-u_{2}(s)\right\|_{X}^{2} \leq-\left(\eta_{1}(s)-\eta_{2}(s), \dot{u}_{1}(s)-\dot{u}_{2}(s)\right)_{X} \quad \text { a.e. } s \in(0, T) .
$$


Let $t \in[0, T]$. Integrating the previous inequality from 0 to $t$ and using (72), we obtain

$$
\begin{aligned}
& \frac{1}{2}\left\|u_{1}(t)-u_{2}(t)\right\|_{X}^{2} \leq-\left(\eta_{1}(t)-\eta_{2}(t), u_{1}(t)-u_{2}(t)\right)_{X}+ \\
& \int_{0}^{t}\left(\dot{\eta}_{1}(s)-\dot{\eta}_{2}(s), u_{1}(s)-u_{2}(s)\right)_{X} d s
\end{aligned}
$$

We deduce that

$$
\begin{aligned}
& C\left\|u_{1}(t)-u_{2}(t)\right\|_{X}^{2} \leq\left\|\eta_{1}(t)-\eta_{2}(t)\right\|_{X}\left\|u_{1}(t)-u_{2}(t)\right\|_{X}+ \\
& \int_{0}^{t}\left\|\dot{\eta}_{1}(s)-\dot{\eta}_{2}(s)\right\|_{X}\left\|u_{1}(s)-u_{2}(s)\right\|_{X} d s .
\end{aligned}
$$

Using Young inequality, we get

$$
\begin{aligned}
& \left\|u_{1}(t)-u_{2}(t)\right\|_{X}^{2} \leq C\left(\left\|\eta_{1}(t)-\eta_{2}(t)\right\|_{X}^{2}+\right. \\
& \int_{0}^{t}\left\|\dot{\eta}_{1}(s)-\dot{\eta}_{2}(s)\right\|_{X}^{2} d s+\int_{0}^{t}\left\|u_{1}(s)-u_{2}(s)\right\|_{X}^{2} d s .
\end{aligned}
$$

On the other hand, as

$$
\eta_{1}(t)-\eta_{2}(t)=\int_{0}^{t}\left\|\dot{\eta}_{1}(s)-\dot{\eta}_{2}(s)\right\|_{X}^{2} d s
$$

we can obtain

$$
\left\|\eta_{1}(t)-\eta_{2}(t)\right\|_{X}^{2} \leq C \int_{0}^{t}\left\|\dot{\eta}_{1}(s)-\dot{\eta}_{2}(s)\right\|_{X}^{2} d s
$$

Using now (92) in (91), we have then

$$
\left\|u_{1}(t)-u_{2}(t)\right\|_{X}^{2} \leq C\left(\int_{0}^{t}\left\|\dot{\eta}_{1}(s)-\dot{\eta}_{2}(s)\right\|_{X}^{2} d s+\int_{0}^{t}\left\|u_{1}(s)-u_{2}(s)\right\|_{X}^{2} d s\right) .
$$

Taking into account the Gronwall's inequality we deduce

$$
\left\|u_{1}(t)-u_{2}(t)\right\|_{X}^{2} \leq C \int_{0}^{t}\left\|\dot{\eta}_{1}(s)-\dot{\eta}_{2}(s)\right\|_{X}^{2} d s
$$

which yield

$$
\int_{0}^{t}\left\|u_{1}(s)-u_{2}(s)\right\|_{X}^{2} d s \leq C \int_{0}^{t}\left\|\dot{\eta}_{1}(s)-\dot{\eta}_{2}(s)\right\|_{X}^{2} d s
$$


From (90), (93) and (94) we obtain

$$
\left\|\Lambda \eta_{1}(t)-\Lambda \eta_{2}(t)\right\|_{X}^{2} \leq C \int_{0}^{t}\left\|\dot{\eta}_{1}(s)-\dot{\eta}_{2}(s)\right\|_{X}^{2} d s .
$$

Iterative the last inequality $m$ times we infer

$$
\left\|\Lambda^{m} \eta_{1}(t)-\Lambda^{m} \eta_{2}(t)\right\|_{X}^{2} \leq C^{m} \int_{0}^{t} \int_{0}^{s_{1}} \cdots \int_{0}^{s_{m-1}}\left\|\dot{\eta}_{1}\left(s_{m}\right)-\dot{\eta}_{2}\left(s_{m}\right)\right\|_{X}^{2} d s_{m} \cdots d s_{1},
$$

where $\Lambda^{m}$ denotes the power of the operator $\Lambda$. The last inequality give

$$
\left\|\Lambda^{m} \eta_{1}(t)-\Lambda^{m} \eta_{2}(t)\right\|_{W^{1,2}(0, T ; X)}^{2} \leq \frac{C^{m} T^{m}}{m !}\left\|\eta_{1}-\eta_{2}\right\|_{W^{1,2}(0, T ; X)}^{2},
$$

which implies that $m$ sufficietly large power $\Lambda^{m}$ of $\Lambda$ is a contraction in the Banach space, since

$$
\lim _{m \longrightarrow+\infty} \frac{C^{m} T^{m}}{m !}=0
$$

it follows now from Banach's fixed point theorem that there exists a unique element $\eta^{*} \in \mathcal{W}$ such that $\Lambda^{m} \eta^{*}=\eta^{*}$. Moreover, since

$$
\Lambda^{m}\left(\Lambda \eta^{*}\right)=\Lambda\left(\Lambda^{m} \eta^{*}\right)=\Lambda \eta^{*},
$$

we deduce that $\Lambda \eta^{*}$ is also a fixed point of the operator $\Lambda^{m}$. By the uniqueness of the fixed point, we conclude that $\Lambda \eta^{*}=\eta^{*}$, which shows that $\eta^{*}$ is a fixed point, we conclude that $\Lambda \eta^{*}=\eta^{*}$.

\section{- Step 4:}

In the fourth and last step of our demonstration, we have now all the ingredients to provide the proof of the Theorem 2 :

\section{Existence}

Let $\eta^{*} \in W^{1,2}(0, T ; V)$ be the fixed point of the operator $\Lambda$, and let $u_{\eta^{*}}, \varphi_{\eta^{*}}$ be the solutions of problems $\mathcal{P} \mathcal{V}_{\eta}^{1}$ and $\mathcal{P} \mathcal{V}_{\eta}^{2}$, respectively, for $\eta=\eta^{*}$. It follows from (87) that

$$
\left(\eta^{*}(t), v\right)_{V}=\int_{0}^{t} \theta(t-s) u_{\eta} *(s) d s+a_{e}^{*}\left(\varphi_{\eta} *(t), w\right) \quad \forall v \in V, t \in[0, T]
$$

and, therefore, (71), (72) and (83) imply that $\left(u_{\eta^{*}}, \varphi_{\eta^{*}}\right)$ is a solution of problem $\mathcal{P V}$. Regularity (69) of the solution follows from Lemmas 3 and 5. 


\section{Uniqueness}

The uniqueness of the solution follows from the uniqueness of the fixed point of the operator $\Lambda$. It can also be obtained by using arguments similar as those used in $[1,2]$.

\section{Acknowledgments}

The authors would like to thank both reviewers for their insightful comments of the paper, as thses comments led us to an improvement of the work. Our revisions refelct all reviewers "suggestions, corrections and readers" comments.

\section{References}

[1] M. Dalah, Analyse of a Electro-Viscoelastic Antiplane Contact Problem With Slip-Dependent Friction, Electronic Journal of Differential Equations. 161 (2009), 1-16.

[2] M. Sofonea, M. Dalah, Antiplane Frictional Contact of Electro-Viscoelastic Cylinders, Electronic Journal of Differential Equations. 161 (2007), 1-14.

[3] W. Han and M. Sofonea, Quasistatic Contact Problems in Viscoelasticity and Viscoplasticit, Studies in Advanced Mathematics, Americal Mathematical Society, Providence, RI-International Press, Somerville MA. 30 (2002).

[4] C. O. Horgan, Antiplane shear deformation in linear and nonlinear solid mechanics, SIAM Rev. 37 (1995), 53-81.

[5] M. Sofonea, M. Dalah and A. Ayadi, Analysis of an antiplane electro-elastic contact problem, Adv. Math. Sci. Appl. 17 (2007), 385-400. 